\title{
FULMINANT DIFFUSE SYSTEMIC SCLEROSIS FOLLOWING AUTOLOGOUS STEM-CELL TRANSPLANTATION IN A PATIENT WITH MULTIPLE MYELOMA
}

Júlia Boechat Farani ${ }^{1, \star}$, Erika Biegelmeyer ${ }^{1}$, Evelise Mileski do Amaral Berlet ${ }^{1}$, Marcel Mathias Villaça ${ }^{1}$, Marília Voges de Souza ${ }^{1}$, Mauro Waldemar Keiserman ${ }^{1}$

1.Pontifícia Universidade Católica do Rio Grande do Sul, Porto Alegre (RS), Brazil.

*Corresponding author: juliaboechat@globo.com

\section{BACKGROUND}

Autologous stem-cell transplantation (ASCT) is considered an investigational therapy for severe systemic sclerosis (SSc). However, it might not prevent relapsing disease on predisposed patients.

\section{CASE REPORT}

A 67-year-old white male patient was admitted to our hospital in April 2021, complaining of progressive dyspnea and diffuse skin thickening. He had a previous diagnosis of systemic sclerosis (SSc) sine scleroderma since 2016, initially presenting with Raynaud's phenomenon, esophageal hypomotility, as well as dyspnea due to pulmonary arterial hypertension (PAH), with an estimated pulmonary artery systolic pressure (PASP) of $98 \mathrm{mmHg}$ confirmed by right cardiac catheterization and treated with sildenafil and ambrisentan. Back then, cutaneous manifestations were absent, and investigation revealed a positive antinuclear antibody (ANA) of 1:160 with a centromeric (speckled) pattern, suggestive of anticentromere antibodies. A monoclonal protein was also detected upon laboratory workup, leading to hematological investigation and a concurrent diagnosis of multiple myeloma (MM) in 2017. Induction chemotherapy was performed with bortezomib, cyclophosphamide and low-dose dexamethasone, followed by stem-cell collection and a successful ASCT. He went through pulmonary aspergillosis as a post-transplantation complication, but the outcome was favorable, with complete remission of both MM and SSc during the next four years, as well as PASP normalization and no need for further immunosuppressive therapy. However, in January 2021, there was relapsing of dyspnea on mild exertion, as well as facial and trunk telangiectasias and diffuse sclerotic lesions starting as erythematous plaques with intense pruritus and progressing to hyperpigmented and depigmented atrophic skin areas ("salt-and-pepper" lesions). COVID-19 infection was ruled out. There was chronic hypoxemia and laboratory signs of relapsing MM, with anemia, renal insufficiency, and a rise in paraproteins. Hematological treatment was resumed with lenalidomide. Cutaneous biopsy revealed mild dermal sclerosis suggestive of scleroderma. Chest CT revealed an increase in the pulmonary artery trunk and pulmonary congestion, without fibrosis. Echocardiogram showed left ventricular diastolic dysfunction with overload of right cavities and severe PAH with a PASP of $106 \mathrm{mmHg}$. He was treated with pulse methylprednisolone 500 mg daily for 3 days, followed by cyclophosphamide and oral prednisone, as well as loop diuretics, domperidone, sildenafil and ambrisentan, with initial cutaneous improvement and pulmonary stabilization. Unfortunately, he died one month later due to sepsis.

\section{CONCLUSION}

This is an intriguing case of severe relapsing SSc four years after ASCT as a treatment for concurrent MM. It is possible that relapsing MM might have induced this fulminant course of the preexisting autoimmune disease.

\section{KEYWORDS}

Systemic sclerosis, Autologous stem-cell transplantation, Multiple myeloma. 